\title{
Application of Open Source Image Guided Therapy Software in MR-guided Therapies
}

\author{
Nobuhiko Hata ${ }^{1}$, Steve Piper ${ }^{2}$, Ferenc A Jolesz ${ }^{1}$, Clare MC Tempany ${ }^{1}$, \\ Peter McL Black ${ }^{2}$, Shigehiro Morikawa ${ }^{3}$, Horoshi Iseki ${ }^{4}$, Makoto Hashizume ${ }^{5}$, \\ and Ron Kikinis ${ }^{1}$ \\ ${ }^{1}$ National Center for Image-guided Therapy, Department of Radiology, \\ Brigham and Women's Hospital and Harvard Medical School \\ hata@bwh. harvard.edu \\ ${ }^{2}$ Department of Neurosurgery, Brigham and Women's Hospital \\ and Harvard Medical School \\ ${ }^{3}$ Shiga Medical University \\ 4 Tokyo Women's Medical University \\ ${ }^{5}$ Kyusyu University ${ }^{\star}$
}

\begin{abstract}
We present software engineering methods to provide free open-source software for MR-guided therapy. We report that graphical representation of the surgical tools, interconnectively with the tracking device, patient-to-image registration, and MRI-based thermal mapping are crucial components of MR-guided therapy in sharing such software. Software process includes a network-based distribution mechanism by multi-platform compiling tool CMake, CVS, quality assurance software DART. We developed six procedures in four separate clinical sites using proposed software engineering and process, and found the proposed method is feasible to facilitate multicenter clinical trial of MR-guided therapies. Our future studies include use of the software in non-MRguided therapies.
\end{abstract}

\section{Introduction}

Among image-guided therapy (IGT) tools, MR-guided therapy is a widely used option as evidenced by the more than 400 articles published in a literature database. We have even observed the acceleration of its prevalence since clinicians started to use diagnostic close-bore scanners in surgery.

However, the active development of new MR-guided therapy option is restricted in the scope of procedures, sites of implementation, and most importantly lack of flexibility in enabling software tools. The long-term goals of improving interventional and surgical procedures and attendant outcomes, reducing costs, and achieving broad utilization of software tools can be achieved by identifying the commonality of the enabling software and developing a software framework that can dynamically accommodate the specific needs of applications.

\footnotetext{
* Supporting grant: 5P41RR013218, 5P01CA067165,5U41RR019703, 5R01CA109246, 1R01CA111288, 5U54EB005149 from NIH, NSF 9731748, CIMIT, METI.
} 
Such software should be open-source and proprietary free so that the openness lets programmers obtain, critique, use, and build upon the source code without licensing fees [1]. A similar model for sharing software has been actively developed in bioinformatics domain $[2,3]$. The open source software for bioinformatics has made it easier to create customized pipelines or analysis in gene expression study, or tailored medicine. There is no question that bioinformatics has embraced open source software and has been benefited from them in developing new methods.

Our objective in this paper is to present software engineering methods to share the commonality of software tools and provide robust testing, documenting, and version control mechanism in computer-enhanced MR-guided therapies. We refined these engineering methods over 2,000 cases of MR-guided brain, prostate, and liver therapies in four separate clinical centers by sharing the software platform but developing unique clinical applications in each centers. We used the 3D Slicer (www.slicer.org) originally developed for medical image processing, but modified to fit the needs of MR-guided therapy for this study. Our engineering challenge was to find the most commonality among the applications in different sites, while ensuring the flexibility to develop unique IGT application in those site with minimum overhead. The methods presented here contain summery of commonality we found over the course of clinical cases as well as unique enabling tools for each site's application.

\section{Extending Medical Image Processing Software for MR-guided Therapy}

We extended 3D Slicer [4] for MR-guided therapy to develop enabling software for MR-guided ablation therapy at four clinical sites. The 3D Slicer is freely available, open-source software for visualization, registration, segmentation, and quantification of medical data for diagnostic purposes. In addition to native tools originally developed for medical image processing, we developed tools and visualization methods useful for MR-guided therapies.

Fiducial/Locator. We prepared common graphical representation of tools specific to MR-guided therapy. Those models include fiducial points with arbitrarily changeable diameter and the locator representing the tracking wand. The locator model is mostly used to represent the digitally tracked wand; in-bore MR-guided surgery uses the optical tracking sensor placed over the area, and out-of-bore MR-guided surgery uses an optical tracking sensor placed outside the bore. In addition to mouse-controlled slice selection, the 3D Slicer provides slice selection using the locator, i.e. digital wand; this is the most common use of a digital wand in navigation software during surgery.

Users can define fiducial points in the three-dimensional space in both $2 \mathrm{D}$ image planes and 3D graphics scenes using a mouse pointer or digital wand. For instance, Morikawa et al. used fiducials to mark an ablated lesion in a liver during MR-guided microwave therapy [5]; in brain surgery at Brigham and Women's 
Hospital, we used the fiducials for marking a cortical stimulation point on the cortex and compared them with the images.

Interconnectivity to Tracking Device. The 3D Slicer communicates with an external device in various formats. A relatively simple TCP/IP-based client/ server communication package has been in use in MR-guided interventions to retrieve updated images and the coordinates of digital wands[6]. A similar approach was used in linking the 3D Slicer to commercial navigation software, in which the coordinates of the tracker are shared between the two. In one study at Brigham and Women's Hospital, the optical tracking sensor is directly accessed from a module of the 3D Slicer, without client/server communication [6].

Patient-to-image Registration. The 3D Slicer provides patient-to-image registration using fiducial markers; a set of fiducials digitized by an external sensor is coupled with image-based digitization to estimate conversion matrix from physical (patient)-coordinate system to image coordinate system. The 3D Slicer provides digitization tools both for the external sensors and images using fiducial markers as the basis for user interaction.

Native image-to-image registration can also be used in MR-guided therapy by fusing a preoperative information-rich image to an intraoperative image, or by fusing the sequence of intraoperative images. Note that the users can bypass the fiducial placement if the optical tracking sensor is calibrated to the imager and confined in the image coordinate system.

Thermal Mapping. MRI can provide a thermal map for ablation therapy and monitor the extent of heat perfusion during microwave or laser-ablation therapy. The combination of stereotactic guidance using preoperative and preprocedural images and thermal mapping is useful to control the ablation therapy and maximize the coverage of an ablation lesion and lessen damage to surrounding critical tissue.

The 3D Slicer provides phase differential computation using a sequence of fast 2D spoiled gradient-recalled sequences as inputs. The magnitude, phase, real, and imaginary images can be transferred online in real time from the MRI scanner to the 3D Slicer workstation and presented to the physicians.

\section{Software Process}

Cross-platform Development. The 3D Slicer was developed using a crossplatform environment, CMake. CMake provides compilation management for Windows, Unix, and Mac OS platforms[7]. This multiplatform support was crucial to provide options for the IGT computer platform depending on the needs and requirements of the project. In our clinical applications, we used the Linux platform as well as Windows.

Software Distribution. We provided the software tools to facilitate their distribution to the collaborating institutions and researchers. Central to this effort 
were concurrent version systems (CVS), which records the history of sources files. A client/server version of CVS enables developers separated by geographic distance to function as a team. Documentation generation tools were Doxygen and LaTeX to produce online manual pages.

Quality Assurance. DART server was employed in the 3D Slicer for quality assurance in multiple projects. In addition, we enhanced DART to provide historical reporting, trending, and analysis of test results. DART currently supports results transmission using HTTP and FTP protocols. Regular testing is performed at scheduled intervals, often nightly, and it collects the overall software development activities for the past 24 hours. On-demand testing allows each developer to make local changes and immediately evaluate them against the established gold standard.

\section{Results}

The software we developed has been applied to multicenter clinical studies for MR-guided therapy at four different sites. Each center had one or two software engineers developing modules for clinical applications. Each center was also responsible for obtaining Institutional Review Boards' approval on human subject studies. The version of Slicer used in each site varies depending on the start of the clinical studies. In the following sections, we report the nature of the clinical application, number of cases, and unique function added. We found that average man-hours invested to develop new procedures were 286 man-hours and average number of codes per procedure was 2446 .

\subsection{MR-guided Brain Tumor Surgery}

We used the 3D Slicer (version 2.0 - 2.6) to register serial intraoperative MRIs and for navigation [6]. The MRI scanner used was $0.5 \mathrm{~T}$ vertical open magnet (Signa SP/i, GE Healthcare, London, UK). We performed 1,026 craniotomies, 336 biospies, 37 pituitary tumor cases, and 16 cyst drainage cases in MR-guided neurosurgery. Of these, 150 were performed using the 3D Slicer developed and controlled in the software engineering process discussed above. The estimated man-hours needed for this implementation was 160 man-hours and total number of lines in Tcl/Tk and shell script codes for on-line image transfer and tracker data control was 232 .

Figure 1 highlights from the MR-guided brain tumor suregery.

\subsection{In-Bore Prostate Biopsy}

We used the 3D Slicer (version 2.6) for both MR-guided prostate brachytherapy in an open-bore MRI scanner as well as MR-guided prostate biopsy. We had 448 brachytherapy cases and 72 biopsy cases. The 3D Slicer has played a different role in each procedure. MRI scanner used was $0.5 \mathrm{~T}$ vertical open magnet (Signa SP/i, GE Healthcare, London, UK). 

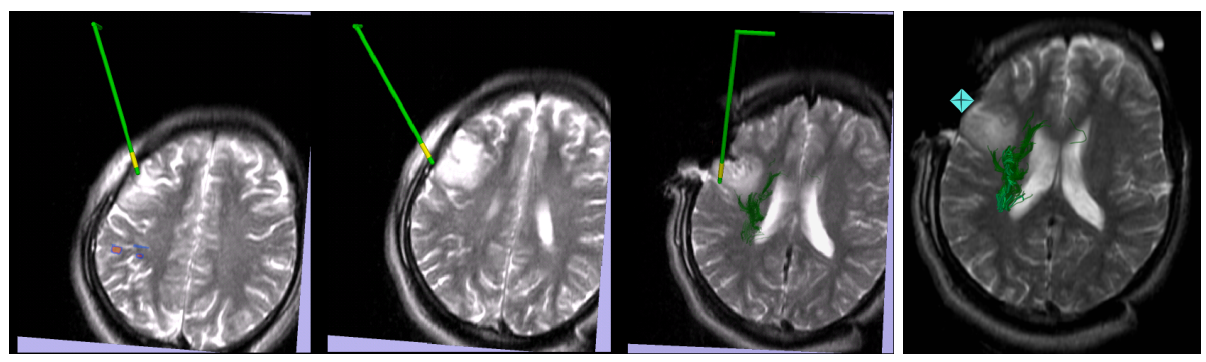

Fig. 1. 22-y-o male with approximately $3 \mathrm{~cm}$ x $3 \mathrm{~cm}$ left posterior frontal tumor. (Three images from far left) the 3D Slicer was helpful in confirming that tumor volume in the resection is not part of the active lesion in fMRI and DTI fused with intraoperative T2 MRI. Images are snapshots from physicians confirmation process using fMRI (shown as a red blob in the far left image), tumor-highlighted lesion (shown as a high-intensity lesion in second and the third images from left). The right image shows the craniotomy and the partially resected tumor. (Far right) The 3D Slicer was helpful in confirming the location of the Ojemann stimulator (blue dot) with respect to the lesion (in T2 image) and the margin.

The 3D Slicer is essential to the MR-guided prostate biopsy. The coordinates of suspicious tumor foci are specified in 3D Slicer and corresponding holes in the needle guiding template grid are computed, which effectively shortens surgery time and reduces the potential for computational errors. A key feature of the system is volumetric data fusion, allowing for target planning on highresolution preoperative $\mathrm{T} 2$-weighted images mapped onto intraoperative $0.5 \mathrm{~T}$ images. Patient-to-image registration methods was modified and used to calibrate the needle guiding template to MRI scanner's coordinate system. The template was calibrated to the MRI scanner using the optical tracking system; and 3D Slicer performed this operation by using both interconnectivity function to the tracker and the patient-to-image registration tool. The prostate biopsy module needed new development for real-time image transfer and scanner control, of which image transfer was already available from neurosurgical applications. Therefore, approximate man-hours invested was 320 man-hours and line of codes added was 3948 lines.

\subsection{In-Bore Laser Ablation Therapy of Brain Tumor}

The 3D Slicer (version 2.6) enabled software for monitoring laser-inducted interstitial therapy (LITT) of a brain tumor in intraoperative MRI. The 3D Slicer was useful to ablate the tumor with integrated tumor segmentation and thermal monitoring tools, processing the intraoperative MR imagery online and in real time. MRI scanner used was $0.5 \mathrm{~T}$ vertical open magnet (Signa SP/i, GE Healthcare, London, UK).

The 3D Slicer, modified specifically for LITT, gave us options to objectively measure the extent of a tumor by image segmentation, mark the critical volume 


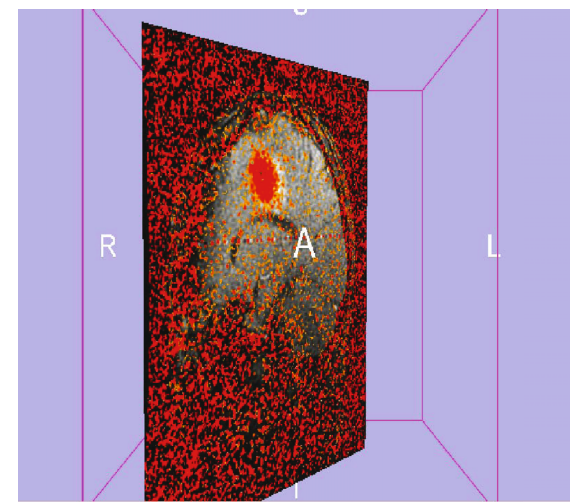

Fig. 2. A snap shot from In-bore laser ablation therapy of brain tumor showing the thermal image (in reddish color) overplayed on the T1-weighted MRI. The procedure uses shared tools of the thermal imaging, image transfer, image registration, and tracking and guidance using in-bore tracking system.

by thermal imaging, and update the treatment plan by comparing the original tumor lesion and treated lesion. Both the original tumor lesion and the ablated lesion were mapped onto the T1-weighted MRI. This overlapped mapping was then used to update the treatment plan, i.e., additional laser firing or repositioning of the fiber, to best cover the tumor with thermal ablation. Figure2 demonstrates an highlights from the In-bore laser ablation therapy of brain tumor.

\subsection{In-Bore Microwave Ablation Therapy of Liver Tumor}

The team at Shiga Medical University, Shiga, Japan, used the 3D Slicer (version 2.2) in a similar manner as in the prostate biopsy above. The 3D Slicer could display an intraprocedural thermal MR image in combination with two reformatted images from preoperative high-resolution 3D volume data [7].

A unique innovation was the foot-printing, which recorded and displayed football-shaped coagulated areas of $20 \mathrm{~mm}$ in diameter and $30 \mathrm{~mm}$ in length along the electrode in the 3D space. The direction of the electrode was obtained from the optical tracking system information. The footprint was placed along the direction of the electrode to record the "coagulated area", was helpful to optimally cover the large tumor by placing electrode in multiple place inside the tumor. The number of man-hours spent on this development was 120 man-hours, and line of codes was 1659 .

\subsection{Slide-in-and-Out Ablation Therapy of Liver Tumor}

In 11 Percutaneous Ethanol Injection Therapy (PEIT) and 3 Radiofrequency Ablation (RFA) interventions in Kyusyu University, Fukuoka, Japan, 3D Slicer (version 2.2) with intraoperative MR images provided useful information in de- 
termining the needle orientation and placement for the target tumors. MRI scanner used was low-field horizontal open magnet (Hitachi AIRIS-II, Tokyo, Japan).

By attaching the markers of optical position sensor to an ultrasound needle insertion adapter, a virtual needle path was intraoperatively displayed with tumor and vessel models constructed from segmentation. The reconstructed cross sectional MR images identical to ultrasound images including the needle positions were also provided after point-based MR registration with patient body using MRI visible markers. This MR based navigation provided physicians with strong confidence when the tumors were not clearly identified in conventional ultrasound guided procedures [8]. We observed deformation of liver which then led to discrepancy between MRI and ultrasound images. Approximately 480 man-hours were spent on this project.

\subsection{Slide-in-and-Out Brain Tumor Surgery with Commercial Navigation Software}

The team at Tokyo Women's Medical University, Tokyo, Japan have used the 3D Slicer, as navigation software in their MR-guided navigation therapy. The 3D Slicer was linked to a commercial neuro-navigation system (experimental product, Toshiba, Tokyo, Japan). MRI scanner used was low-field horizontal open magnet (Hitachi AIRIS-II, Tokyo, Japan).

Tumor segmentation method was developed and implemented in 3D Slicer. 3D Slicer's unique role in this study was transfer intraoperative images from the scanner and perform tumor segmentation followed by volume measurement. 3D slicer was set up next to the commercial neuro-navigation system and received tracker coordinates and patient-to-image registration using fiducial markers. The role of the 3D Slicer was complementary to the commercial navigation system, which doesnt provide interface to state-of-the-art segmentation, volume measurement, and visualization. Approximately 320 man-hours were spent on this project.

\section{Discussion}

We presented a software engineering methods to provide free open-source software for MR-guided therapy. We found that graphical representation of the surgical tools, interconnectively with the tracking device, patient-to-image registration, and MRI-based thermal mapping are crucial components of MR-guided therapy in sharing software. We developed six procedures in four separate clinical sites using proposed softare process, and found the method is feasible to facilitate multicenter clinical trial of MR-guided therapies.

There are surprisingly few efforts to share software resources in image-guided therapy; among them is Image Guided Surgery Toolkit (IGSTK) [9]. The toolkit contains the basic software components to construct an image-guided system, including a tracker and a default graphical user interface that includes a fourquadrant view with image overlay. Our solution is complementary to IGSTK; we 
have been trying to bridge medical image processing and image-guided surgery, yet requirements for the IGT-specific component are confined to target application. Our emphasis on bridging medical image computing (MIC) and computer assisted intervention (AI) was carried out in this stud. We found that registration and segmentation were the key enabling technology for new procedures.

Our future study include use of the software in non-MR-guided therapies, which should benefit from combined use of medical image processing and IGTspecific tools.

\section{References}

[1] McDonald, C.J., Schadow, G., Barnes, M., Dexter, P., Overhage, J.M., Mamlin, B., McCoy, J.M.: Open Source software in medical informatics-why, how and what. Int. J. Med. Inform. 69, 175-184 (2003)

[2] Marturano, A., Chadwick, R.: How the role of computing is driving new genetics' public policy. Ethics Inf. Technol. 6, 43-53 (2004)

[3] Stewart, J.E., Mangalam, H., Zhou, J.: Open Source Software meets gene expression. Brief Bioinform. 2, 319-328 (2001)

[4] Gering, D.T., Nabavi, A., Kikinis, R., Hata, N., O'Donnell, L.J., Grimson, W.E.L., Jolesz, F.A., Black, P.M., Wells, W.M.: An integrated visualization system for surgical planning and guidance using image fusion and an open MR. Journal of Magnetic Resonance Imaging 13, 967-975 (2001)

[5] Morikawa, S., Inubushi, T., Kurumi, Y., Naka, S., Sato, K., Demura, K., Tani, T., Haque, H.A., Tokuda, J., Hata, N.: Advanced computer assistance for magnetic resonance-guided microwave thermocoagulation of liver tumors. Academic Radiology 10, 1442-1449 (2003)

[6] Nabavi, A., Black, P.M., Gering, D.T., Westin, C.F., Mehta, V., Pergolizzi Jr., R.S., Ferrant, M., Warfield, S.K., Hata, N., Schwartz, R.B., Wells 3rd, W.M., Kikinis, R., Jolesz, F.A.: Serial intraoperative magnetic resonance imaging of brain shift. Neurosurgery 48, 797-798 (2001)

[7] Martin, K., Hoffman, B.: An open source approach to developing software in a small organization. IEEE Software 24, 46 (2007)

[8] Hong, J., Nakashima, H., Konishi, K., Ieiri, S., Tanoue, K., Nakamuta, M., Hashizume, M.: Interventional navigation for abdominal therapy based on simultaneous use of MRI and ultrasound. Med. Biol. Eng. Comput. (2006)

[9] Gary, K., Ibanez, L., Aylward, S., Gobbi, D., Blake, M.B., Cleary, K.: IGSTK: An open source software toolkit for image-guided surgery. Computer 39, 46 (2006) 